\title{
¿SON LOS ALUMNOS CAPACES DE ATRIBUIR A LOS MICROORGANISMOS ALGUNAS TRANSFORMACIONES DE LOS ALIMENTOS?
}

\author{
Díaz GONZÁlEZ, R., LÓPEZ RODRÍGUEZ, R., GARCÍA LOSADA, A., ABUÍN FIGUEIRAS,G., \\ NOGUEIRA ABUÍN, E. Y GARCÍA GANDOY, J.A. \\ Departamento de Didáctica de las Ciencias Experimentales de la Universidad de Santiago. \\ Escuela Universitaria de Magisterio de Lugo. Av. Ramón Ferreiro, 10. Lugo.
}

\section{SUMMARY}

In this paper we investigate if primary school students are able to relate microorganisms with food transformations. The subjects of this study were 3434 th. and 7 th. grade children from Lugo (Spain) and the instrument used consisted of four multiple choice questions. The results shed light on the difficulties students have at these levels when they try to apply their ideas to interpret biological phenomena. Finally, we discuss some implications for Science teaching and learning.

\section{INTRODUCCIÓN}

Las investigaciones que en los últimos años se vienen realizando sobre enseñanza de las ciencias señalan serias dificultades, motivadas entre otras razones por la existencia de ideas previas en los alumnos y por el escaso éxito de algunas estrategias de aprendizaje que se venían utilizando.

Estas concepciones, que no siempre son errores y pueden jugar un buen papel en el individuo ya que contribuyen a que sea asequible la nueva información que de otro modo resultaría ardua, han recibido diferentes nombres: ideas previas, preconcepciones, representaciones, ideas alternativas, etc., denominaciones todas ellas que, como apuntan Pozo y otros (1992), responden a un enfoque conceptual del aprendizaje de las ciencias en el que no se tienen en cuenta estructuras de conocimiento generales, sino unidades de conocimiento específicas, lo cual implicaría un aprendizaje como cambio de ideas aisladas y no de estructuras cognitivas.

Últimamente algunos estudios ponen de manifiesto que las ideas, aunque ligadas frecuentemente al contexto, no son tan aisladas como pudiera parecer, observándose cierto grado de coherencia; coherencia con el estadio piagetiano (Shayer y Adey, 1984; Monk, 1990) y coherencia en cuanto a que una misma idea es aplicada de igual forma en diferentes contextos (Engel Clough y Driver, 1986; Arnay, 1988; Jiménez,1990). Desde otras corrientes también se pretende abanđonar el nivel de especificidad con el que se habia venido trabajando sobre ideas aisladas, para adoptar una posición más estructurada de ideas mantenidas en diferentes dominios, que constituyen por ejemplo las «miniteorías» de Claxton (1987) o "teorias implícitas», o bien los denominados por Engel Clough y Driver «esquemas conceptuales»: explicaciones que se mantienen en más de un contexto.

Haciendo un recorrido por las investigaciones sobre ideas previas en ciencias experimentales, nos encontramos con que las ideas de los estudiantes acerca de conceptos relacionados con microbiología han sido hasta el momento muy poco estudiadas. La mayoría de los trabajos en este campo se centran en el estudio de 
conceptos como salud, enfermedad y prevención (Brumby et al., 1985; Eiser, 1985), siendo más escasas las investigaciones de conceptos como contagio, mohos o microbios en generai (Nagy, 1951, 1952, 1953; Barrio, 1990). Complementariamente, las investigaciones realizadas en otros campos afines como el de la célula (Dreyfus y Jungwirth, 1988,1989 ) ponen de manifiesto las dificultades que encierra el conocimiento de todo aquello que escapa a la percepción y asimismo los abundantes estudios sobre lo que los alumnos consideran que puede estar o no vivo (Lucas et al., 1979; Brumby, 1982; O-Saki y Samiroden, 1990) revelan que un amplio porcentaje de la población de estudiantes en edades semejantes a las de este trabajo no consideran, por ejemplo, que organismos visibles y superiores a los microbios puedan ser seres vivos.

Por otro tado, son conocimientos que a lo largo de la historia han necesitado mucho tiempo y debate científico para llegar a esclarecerse. La creencia sobre generacion espontánea, por ejemplo, que existía ya en la antiguedad, no empieza a carnbiar hasta el siglo XVII y no se desvanece completamente hasta finales del XIX. Por ello no podemos esperar que estos conceptos, si existen en los alumnos, vayan a ser sustituidos fácilmente por los conceptos científicos.

En este trabajo, que forma parte de un estudio más amplio, exponemos cómo ha sido la investigación de dos cuestiones relacionadas con la aplicación del concepto «microbio»:

1) ¿Son capaces los alumnos de atribuir a los microorganismos algunas transformaciones de los alimentos?

2) ¿Con qué procesos familiares relacionan el concepto de fermentación?

$Y$ en este sentido, iniciamos la investigación con las siguientes hipotesis:

1) Existen esquemas alternativos para explicar las transformaciones de los alimentos.

2) Algunos esquemas pueden variar con el contexto o con la redacción de las preguntas.

3) Algunos esquemas alternativos revelarán cierta consistencia.

4) Los cursos estudiados, $4^{\circ}$ y $7^{\circ}$ de EGB, se diferenciarán en el uso de algunos de estos esquemas.

\section{METODOLOGÍA}

Hemos elegido como instrumento de diagnóstico un cuestionario escrito con cuatro preguntas de opción múltiple, cuya elaboración ha constituido la fase final de un proceso o estudio previo, en el que se han tenido en cuenta las fases recomendadas por Treagust (1988):

\section{Definición del contenido}

Etapa en la que identificamos los conocimientos básicos que componen el tópico global en estudio (microbiología) y los relacionamos entre ellos mediante el uso de mapa conceptual (Novak y Gowin, 1988). La validez de contenido del mismo ha sido establecida por el equipo investigador, en el cual no han surgido discrepancias.

Ya que el presente trabajo abarca solamente una pequeña parcela del contenido total establecido, no reproducimos aquí el mapa conceptual, del que sólo se utilizan dos conceptos para preparar las pruebas que se describen más adelante. Estos conceptos son:

- Seres vivos unicelulares que transforman los alimentos con resultado beneficioso (ejemplo: produciendo fermentaciones como la de la leche).

- Seres vivos unicelulares que transforman los alimentos con resultado perjudicial (ejemplo: descomposición $\mathrm{y}$ formación de mohos en los alimentos).

\section{Elección de la muestra}

El criterio seguido para elegir los cursos de EGB objeto de estudio ha sido el de localizar aquéllos que preceden a los años con mayor volumen de enseñanza relacionada con microbiología, ya que lo que nos interesaba era conocer realmente las ideas de los alumnos o sus «teorías infantiles» antes de la instrucción formal. Consultando a profesores de todos los cursos de EGB de tres colegios, acerca de siete aspectos relacionados con la microbiología y su distribución en la enseñanza en cada curso, así como revisando los libros de texto de los mismos cursos usados como apoyo, hemos reunido una amplia información que, ordenada convenientemente, nos ha permitido establecer que, en este caso, los cursos con mayor instrucción en este tópico eran $5^{\circ}$ y $8^{\circ}$ de EGB.

Así pues, nos hemos inclinado finalmente por los cursos de $4^{\circ}$ y $7^{\circ}$ de EGB como cursos de estudio, constituyendo la muestra final 343 alumnos de tres colegios públicos de la ciudad de Lugo, 186 de los cuales correspondian a ocho grupos de $7^{\circ}$ de EGB y 157 a seis grupos de $4^{\circ}$ de EGB (curso académico 1993-94).

\section{Obtención de información acerca de los conocimien- tos de los estudiantes. Primera aproximación}

Con el objeto de diseñar el test diagnóstico de la forma más eficaz, es decir, usando como distractores algunos de los errores más comunes, y ya que la información bibliográfica sobre los mismos era insuficiente, optamos por hacer algunas pruebas piloto sobre posibles errores conceptuales, rastreando algunos campos que se vienen considerando relacionados con los mismos: libros de texto e ideas de la historia. 
Libros de texto. En Ia revisión de los mismos, correspondientes a textos de dos editoriales usadas en los colegios estudiados, en los niveles de $1^{\circ}$ a $8^{\circ}$ de EGB, hemos encontrado que la forma en que se trata la enseñanza de algunos procesos podría estar relacionada con la posible aparición de errores en el tema que a nosotros nos ocupa. En este sentido, se describen a continuación algunas de estas apreciaciones.

Así, por ejemplo, en el proceso de la fermentación se da mucha importancia al aspecto industrial (destacándolo además normalmente de forma muy potente mediante apoyatura gráfica como dibujos y fotos de fábricas o procesos industriales), primándose de forma notoria aspectos de manufacturación sobre otros aspectos claves, como el biológico, que parece incluso en ocasiones no tenerse en cuenta.

Por otro lado, cuando se describen procesos de conservación de los alimentos, con el objeto de prevenir los efectos de la vida saprofita de los microorganismos, prácticamente nunca se relacionan estos procedimientos con la vida microbiana estableciendo una relación de causa-efecto, sino que se suelen describir como simples recetas a seguir.

También, cuando se trata el tema de la contaminación, es destacable la ausencia muy generalizada de alusiones a la contaminación biológica, no existiendo en cambio este vacío por lo que respecta a la contaminación química o física.

Y por lo que se refiere al tratamiento sobre procesos de transformaciones de los alimentos, la notoria falta de explicaciones al respecto y la forma en que se hacen algunas de ellas podrían favorecer que se consideren solamente factores físicos o químicos como responsables únicos de dichas transformaciones.

Ideas de la historia. Aun teniendo en cuenta que no existe un total paralelismo entre el razonamiento espontáneo y la historia de la ciencia (Driver et al., 1989), tratamos de aprovechar la utilidad del mismo, que, como señalan Saltiel y Viennot (1985), puede permitirnos no solo reactivar el conocimiento de la historia partiendo del conocimiento infantil, sino que, a la inversa, podríamos esperar algún beneficio recíproco, extrayendo información sobre el posible pensamiento de nuestros estudiantes a partir de la historia de la ciencia.

En este sentido, y en este tema concreto, podría esperarse o tenerse en cuenta como hipótesis de trabajo que los estudiantes, pensando en términos de generación espontánea, pretendan responsabilizar los alimentos de sus propias transformaciones. Quizá como Ios antiguos espontaneístas pensaban, es decir, creyendo que el alimento «crea» los agentes microbiológicos que luego lo transforman; o quizá esta forma de pensar en un cierto «poder» del alimento les permita admitir una transformación sin seres vivos, transformación que tendría su origen en el propio alimento. Esta idea podría resultar más intuitiva que las de origen biológico, que además son poco estimuladas por los libros de texto.
Podría ser posible también que una cierta forma de pensamiento "preformacionista», les lleve a pensar en los microbios no sólo con una configuración similar a los organismos visibles, sinó incluso con cualidades propias del mundo humano (antropomorfismo).

\section{Obtención de información acerca de los conocimien- tos de los estudiantes. Elaboración de pruebas- sondeo}

Recabando una mayor información sobre errores que nos permita la posterior elaboración del cuestionario, hemos recurrido a dos tipos de pruebas: preguntas abiertas y entrevistas.

Preguntas abiertas. Se han formulado dos cuestiones abiertas (Apéndice I) en test de lápiz y papel, con las que básicamente se buscaba obtener información sobre las siguientes cuestiones:

- Cuál es el pensamiento đe los alumnos sobre la transformación que sufre el pan cuando le sale moho, y qué piensan de la aparición de éste. (Se presentaba además, en el momento de realización del test, una barra de pan visiblemente enmohecida).

- Conocer si tienen ideas sobre «fermentación» y, si es así, de qué naturaleza pueđen ser éstas. (La pregunta en este caso es muy abierta, de forma que, partiendo de una simulación Iúdica, se les plantea la simple existencia real o no del proceso y, en su caso, la definición.

Elaborado el test y consensuado por todo el equipo, fue administrado simultáneamente en los tres colegios en diciembre de 1992, siendo contestado por 168 alumnos de $4^{\circ}$ de EGB y 191 alumnos de $7^{\circ}$ de EGB.

Respecto a la primera cuestión, el examen y analisis de las respuestas nos han permitido una categorización amplia sobre lo que los alumnos piensan del origen y naturaleza del moho (cuestión ésta, objeto de otro estudio). Pero de forma implícita, en las respuestas de esas tres preguntas planteadas en esta parte, afloraban asimismo numerosas ideas sobre la transformación y descomposición del alimento, que por lo general parecen concebidos como un proceso intrínseco de la materia sin que intervengan seres vivos ni de dentro ni de fuera del alimento, y en el cual sólo el paso del tiempo parece ser tenido en cuenta como elemento importante. En algunos casos, sin embargo, se planteaban solamente factores de índole física o química, como responsables únicos del proceso. (Ver ejemplos de respuestas en apéndice 2.)

En cuanto al análisis de la segunda cuestión, hemos advertido que un aito porcentaje de alumnos de ambos cursos parece no tener ninguna idea concreta sobre el proceso de la fermentación. Son alumnos que no contestan, o lo hacen con "no sé», o bien con respuestas que hemos catalogado como similares (por ejemplo, «miraría un diccionario"). Entre los estudiantes que manifiestan alguna idea sobre fermentación, son muy pocos aquéllos que lo hacen de forma correcta o siquiera 
próxima al concepto científico (prácticamente ninguno en el nivel de $4^{\circ}$ de EGB). Abundan en cambio asociaciones incorrectas, que hemos agrupado en las siguientes categorías:

- Ideas relacionadas con hervir: expresan una idea de fermentación relacionada con el proceso de ebullición o características del mismo ("estar agitado», «estar caliente», «cocer», «hervir»...).

- Ideas relacionadas con la germinación. Ejemplos: «el campo fermenta cuando se cultiva»; «las semillas fermentan»; «la fermentación es cuando brota la raíz de la planta».

- Ideas relacionadas con heridas. Ejemplos: «cuando se tiene una herida»; «cuando una herida se infecta»; «cuando en una herida hay porquería».

- Ideas relacionadas con roturas de huesos. Ejemplos: "cuando se rompe un hueso"; "cuando te rompes un tobillo, después fermenta»; "cuando tienen que escayolarte».

Entrevistas individuales. Se ha utilizado el modelo de entrevistas acerca de situaciones cotidianas (Osborne, 1980; Gilbert et al., 1982), durante el cual se presentaba a los alumnos un alimento -un chorizo- que en gran parte de su superficie estaba visiblemente cubierto por moho. El propósito de la entrevista era fundamentalmente obtener una mayor información sobre el pensamiento de los alumnos acerca de las causas de la descomposición y alteración de los alimentos, tratando de esclarecer y conocer con mayor precisión algunos de los conceptos erróneos puestos de manifiesto en la prueba de preguntas abiertas: ¿es un proceso espontáneo que no necesita catusas?; si existen causas, ¿đe qué tipo son: sólo factores físico-químicos..., seres vivos...?

La entrevista ha sido realizada a 14 alumnos de $4^{\circ}$ de EGB y 18 alumnos de $7^{\circ}$ de EGB, durante el tercer trimestre del curso académico 1992-93.

Los resultados, interpretados mediante análisis cualitativo (Burgess, 1985; Bliss et al., 1987), nos han permitido resumir con respecto a esta cuestión la siguiente categorización básica:

1. Proceso que no necesita ninguna causa definida («paso del tiempo», «caducidad»).

\section{Proceso causado por:}

2.1. Factores físicos o químicos («humedad», «polvo», «aire»...).

\subsection{Seres vivos:}

2.2.1. Sólo organismos que están en el suelo («s6lo se pudren las cosas en el suelo»).

2.2.2. Visión antropomórfica («los bichitos actúan sólo sobre alimentos que saben que están abandonados»).
2.2.3. Microorganismos en general («atacan al alimento en cualquier parte").

\section{Elaboración del cuestionario}

Con la información recopilada en las fases anteriormente escritas, nos hemos planteado dos cuestiones:

Cuestión 1. ¿Son los alumnos capaces de atribuir a los microorganismos algunas transformaciones de los alimentos?

Tratamos de obtener respuesta a esta cuestión con las preguntas 1, 2, 3 del cuestionario finalmente elaborado (Apéndice 3). En las preguntas 1 y 2 se prueba el esquema científico o microbiológico (MICR), frente a los demás esquemas alternativos que nos parecen posibles: antropomórfico (ANTR), sin causa definida (SIN C.) y por causas físicas o químicas (F/Q) teniendo en cuenta que, aún cuando éstas últimas son responsables de algunas transformaciones como enranciamientos y otras, no lo son en los dos ejemplos o contextos concretos presentados, aunque pudieran ser las explicaciones preferidas por los alumnos. También se daban en el cuestionario las opciones de respuesta "No sé» y «Otras razones», con el objeto de no forzar la elección de las demás alternativas (éstas dos últimas contestaciones aparecerán en las tablas reflejadas en conjunto, con la abreviatura «OTRA»).

La explicación «Sin causa» era bastante frecuente en preguntas abiertas y la entrevista; no obstante, se pudo constatar que ambas situaciones coexistían con el conocimiento de los microbios e incluso con la capacidad de éstos para «atacar» los alimentos. Por ello, con la pregunta 3 , intentamos saber en qué medida piensan o conocen los alumnos que los microbios «se relacionan" con los alimentos. Utílizamos aquí una redacción en la que se evita describir relaciones concretas (ni formación de moho, ni fermentación...) y con términos muy empleados en fases anteriores por los propios niños, como «atacar» y "comer», los cuales expresan relaciones con el alimento muy activas y quizá algo antropomórficas, en Iugar de las expresiones más científicas y pasivas como "multiplicarse sobre la leche" o "caen en el queso». Además, nos preguntábamos si esta idea de relación con el alimento era una idea amplia, o si por el contrario era una idea limitada por el antropomorfismo («sólo sobre alimentos que saben que están abandonados»), o por conocimientos académicos ( «sólo alimentos en el suelo, por estar allí los microbios descomponedores)), o incluso si esta idea de relación no existe en muchos casos.

Cuestión 2. ¿Con qué procesos familiares relacionan el concepto de fermentación?

Para encontrar soluciones o respuestas a esta cuestión, se elabora la pregunta 4 del cuestionario (Apéndice 3). Básicamente, intentamos ver con esta pregunta, en qué medida los alumnos de $4^{\circ}$ y $7^{\circ}$ de EGB relacionan el 
concepto de fermentación con aquellos procesos donde está presente o es necesario (alternativas u opciones de respuesta: $2,3,7$ ); para lo cual se introducen también, como distractores, otras opciones de respuesta que representan explicaciones directamente relacionadas con las principales ideas alternativas que los mismos alumnos habian manifestado en fases anteriores del estudio (alternativas u opciones: $1,4,5,6$ ).

\section{RESULTADOS Y ANÁLISIS}

Cuestión 1. ¿Son los alumnos capaces de atribuir a los microorganismos algunas transformaciones de los alimentos?

En las tablas I y II y gráficos correspondientes (Figs. 1 y 2), se muestran los porcentajes de estudiantes que mantienen cada uno de los esquemas investigados en las preguntas 1 y 2 del cuestionario y en ambos grupos de edad.

La utilización del esquema aceptado científicamente s6́lo alcanza porcentajes que van del $3 \%$ al $11 \%$, siendo los esquemas alternativos «Sin causa» $\mathrm{y}$ «Factores físicos o químicos", los más elegidos por los estudiantes de ambos cursos.

Tabla I

Pregunta 1: Yogur.

\begin{tabular}{|l|c|c|c|r|l|}
\hline & $4^{\circ}$ curso & $7^{\circ}$ curso & C.Yates & CHI-C & Signiffcac. \\
\hline ANTR. & $11 \%$ & $5 \%$ & & 2,45 & No signif. \\
\hline SIN C. & $30 \%$ & $23 \%$ & & 1,26 & No signif. \\
\hline F/Q & $29 \%$ & $15 \%$ & 4,92 & 5,71 & 0,05 \\
\hline MICR. & $8 \%$ & $11 \%$ & & 0,52 & No signif. \\
\hline OTRA & $22 \%$ & $46 \%$ & & 12,83 & $0,00 \mathrm{I}$ \\
\hline
\end{tabular}

Global $\ldots \ldots \ldots . . .16,57 \ldots \ldots .0,01$

Tabla II

Pregunta 2: Queso.

\begin{tabular}{|l|c|c|c|c|c|}
\hline & $4^{\circ}$ curso & $7^{\circ}$ curso & C.Yates & CHI-C & Significac. \\
\hline ANTR. & $4 \%$ & $1 \%$ & & 1,85 & No signif. \\
\hline SIN C. & $59 \%$ & $39 \%$ & 7,22 & 8,00 & 0,01 \\
\hline F/Q & $29 \%$ & $50 \%$ & 8,37 & 9,23 & 0,01 \\
\hline MICR & $3 \%$ & $4 \%$ & & 0,15 & No signif. \\
\hline OTRA & $5 \%$ & $6 \%$ & & 0,10 & No signif. \\
\hline
\end{tabular}

Giobal ......... $11,70 \ldots \ldots, 05$
Figura 1

Pregunta 1: Yogur.

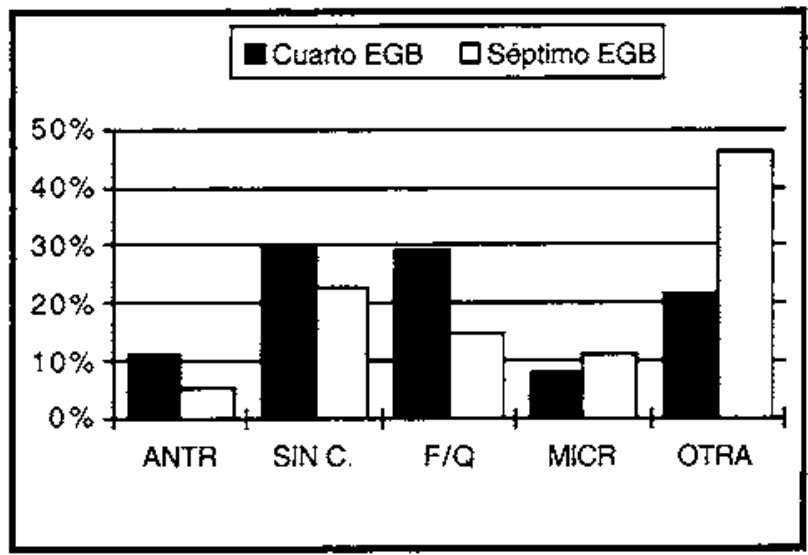

Figura 2

Pregunta 2: Queso.

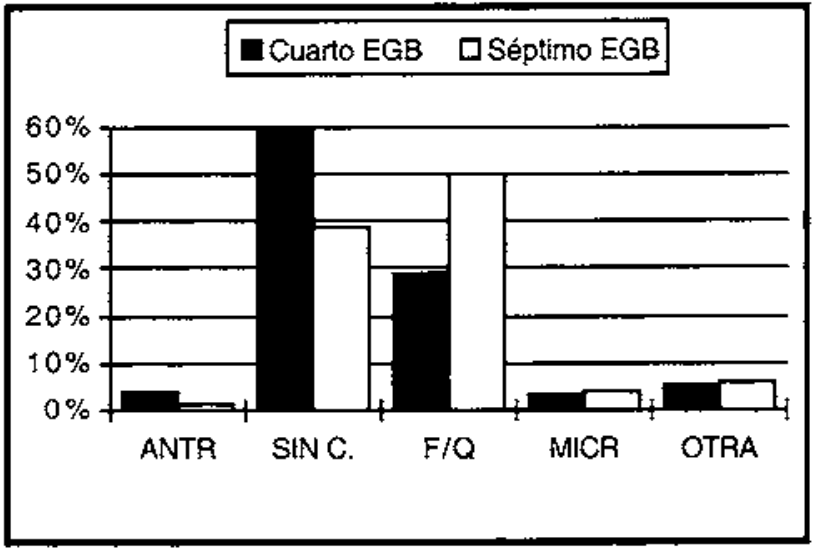

El esquema "Antropomóffico», como era de esperar, es más abundante en $4^{\circ}$ curso que en $7^{\circ}$ curso, sin que esta diferencia entre ambos sea significativa. Una regla de inferencia en el pensamiento causal cotidiano, como puede ser la de semejanza causa-efecto (Pozo, 1987), podría activar modelos analógicos y ser el origen de las ideas antropocéntricas con las que el niño atribuye, a la realidad desconocida (en este caso microscópica), características de una realidad más conocida comoes la del comportamiento humano.

El esquema alternativo "Sin causa» resulta más elegido en el problema del queso que en el del yogur. Quizá en este último les cueste más trabajo admitir que la leche cambie sola, pero no por la necesidad de que concurran seres vivos en el proceso, sino posiblemente por la necesidad de que intervenga mano de obra, ya que en las explicaciones formuladas libremente en la última ailternativa numerosas de ellas (sobre todo de alumnos $\mathrm{co}^{-}$ rrespondientes a $7^{\circ}$ curso) se planteaban en este sentido: 
atribuir este proceso a un simple resultado de manufacturación, normalmente realizado en fábricas.

En cuanto a la diferencia entre ambos cursos, si tomamos las cinco alternativas globalmente, podemos decir que su dependencia de la edad (cursos de $4^{\circ}$ y $7^{\circ}$ ) resulta significativa en las dos preguntas, contexto yogur y contexto queso, para las cuales se obtienen valores de CHI-C, de 16,57 y 11,70 respectivamente, que para cuatro grados de libertad revelan diferencias significativas más allá del nivel 0,02 .

Sin embargo podemos ver en las tablas I y II que analizando esquema por esquema en tabla $2 \times 2$, sólo resultan diferentes ambos cursos para eI esquema «Sin causa» en el problema del queso, y para el esquema «F/Q la correccion de Yates para valorar el nivel de significación, en aquellos casos en que éste puede ser dudoso). El porcentaje de alumnos que utilizan el esquema $« F / Q »$ se mantiene constante en $4^{\circ}$ curso en los dos problemas presentados, mientras que en $7^{\circ}$ curso aumenta notablemente al pasar de yogur a queso. Todo parece indicar que a partir de $4^{\circ}$ curso se van abandonando las explicaciones «Sin causa», para buscar explicaciones «Físicas o químicas», o bien "Otras» explicaciones, pero no para aumentar las «Microbiológicas», al menos significativamente.

La dependencia entre esquemas y contextos a nivel de curso, podemos conocerla examinando las tablas III y IV, referidas a número de alumnos.

Tabla III

Alumnos de $4^{\circ}$ curso. Preguntas 1 y $2 ; n=157$.

\begin{tabular}{|l|c|c|c|c|c|}
\hline & Yogur & Queso & C.Yates & CHI-C & Significac. \\
\hline ANTR. & 18 & 6 & 5,73 & 6,50 & 0,05 \\
\hline SIN C & 48 & 93 & & 26,07 & 0,001 \\
\hline F/Q & 45 & 45 & & 0,00 & No signif. \\
\hline MICR. & 12 & 5 & & 3,05 & No signif. \\
\hline OTRA & 34 & 8 & & 18,58 & 0,001 \\
\hline
\end{tabular}

Global ........ 39,34 ....0,001

Excepto para el esquema científico o microbiológico (MICR) y para el esquema alternativo $« F / Q »$, ambos en $4^{\circ}$ curso, todas las demás explicaciones resultan dependientes del contexto en mayor o menor grado de significación. De todas formas, no sería conveniente sacar conclusiones definitivas en este sentido, ya que el problema presentado, si bien es el mismo a nivel causal (ambos tienen causas microbiológicas), en sus manifestaciones son diferentes (formación de yogur, formación de moho), por lo que, hasta cierto punto, el percibirlos como problemas distintos, podría no indicar solamente dependencia del contexto.
Tabia IV

Alumnos de $7^{\circ}$ curso. Preguntas 1 y $2 ; n=186$.

\begin{tabular}{|l|c|c|c|c|c|}
\hline & Yogur & Queso & C.Yates & CHI-C & Significac. \\
\hline ANTR. & 10 & 1 & 6,15 & 7,79 & 0,05 \\
\hline SIN C. & 42 & 72 & & 10,51 & 0,01 \\
\hline F/Q & 28 & 93 & & 49,94 & 0,001 \\
\hline MICR. & 21 & 8 & 5,8 & 6,64 & 0,05 \\
\hline OTRA & 81 & 12 & & 70,54 & 0,001 \\
\hline
\end{tabular}

Global ....... 107,17 ....... 0,001

Sin embargo, sí creemos que podría ser interesante intentar sacar conclusiones en sentido contrario, esto es, intentar confirmar si el que haya alumnos para los que no se manifiesta dependencia del contexto, sería indicativo de que son consistentes en el esquema que utilizan. Para conocer mejor este último aspecto, es decir, en que medida son consistentes en el uso individual de los esquemas, se han elaborado las tablas de contingencia para los dos problemas en $4^{\circ}$ curso y $7^{\circ}$ curso (tablas $V$ y VI).

Los coeficientes de contingencia obtenidos indican un bajo nivel de consistencia en el uso de los esquemas alternativos y científico en ambos contextos: yogur $y$ queso.

Tabla V

Alumnos de $4^{\circ}$ curso. Preguntas 1 y 2.

\begin{tabular}{|l|c|c|c|c|c|}
\hline Pág. I y 2 & MICR. & SIN C. & F/Q & OTRA & TOTAL \\
\hline MICR. & 0 & 6 & 3 & 3 & 12 \\
\hline SIN C. & 2 & 31 & 12 & 3 & 48 \\
\hline F/Q & 0 & 24 & 17 & 4 & 45 \\
\hline OTRA & 3 & 32 & 13 & 4 & 52 \\
\hline TOTAL & 5 & 93 & 45 & 14 & 157 \\
\hline
\end{tabular}

CHI-C $=9,55 /$ Coefic. Contingencia. $=0,24$ (para yogur y queso) .

Tabia VI

Alumnos de $7^{\circ}$ curso. Preguntas 1 y 2.

\begin{tabular}{|l|c|c|c|c|c|}
\hline Pág. I y 2 & MICR. & SIN C. & F/Q & OTRA & TOTAL \\
\hline MICR. & 3 & 4 & 13 & 1 & 21 \\
\hline SIN C. & 0 & 22 & 19 & 1 & 42 \\
\hline F/Q & 1 & 12 & 14 & 1 & 28 \\
\hline OTRA & 4 & 34 & 47 & 10 & 95 \\
\hline TOTAL & 8 & 72 & 93 & 13 & 186 \\
\hline
\end{tabular}

$\mathrm{CHI}-\mathrm{C}=15,55 /$ Coefic. Contingencia. $=0,28$ (para yogur y queso). 
De todas formas, estos coeficientes, que son una medida de la consistencia general, enmascaran algunas desiguaidades en los datos. En efecto, una observación detallada de las tablas permite constatar que, si bien la no consistencia es notoria en el uso del esquema aceptado científicamente, no ocurre lo mismo con los dos esquemas alternativos más elegidos ( $\mathrm{F} / \mathrm{Q}$ " y "Sin causa»). Así, por ejemplo, en $4^{\circ}$ curso, de 48 alumnos que usan el esquema "Sin causa» en el contexto yogur, 31 emplean de nuevo el mismo esquema en el contexto queso, lo cual supone porcentualmente que en torno al $65 \%$ de estudiantes de este nivel que usan este esquema en el primer caso o contexto mantienen la misma explicación en el segundo; y también podemos comprobar que esta consistencia sólo baja a un $52 \%$ en ei caso de los alumnos de $7^{\circ}$ curso, en esta misma situación.

Tabla VII

Pregunta 3

\begin{tabular}{|l|c|c|}
\hline Explicación & $4^{\circ}(\mathrm{n}=157)$ & $7^{\circ}(\mathrm{n}=186)$ \\
\hline MICR. & $37 \%$ & $49 \%$ \\
\hline ANTR. & $19 \%$ & $8 \%$ \\
\hline SUELO & $24 \%$ & $12 \%$ \\
\hline NUNCA & $12 \%$ & $10 \%$ \\
\hline OTRA & $8 \%$ & $21 \%$ \\
\hline
\end{tabular}

Estos datos, aunque no suficientemente significativos, resultan un indicio para pensar en dos ideas que posiblemente no sean simples nociones dependientes del contexto, ya que, como señalan Engel Clough y Driver (1986); simplemente una consistencia algo mayor que la obtenida en este estudio les daría o podría darles la categoría de teoría informal para el individuo, teoría que guiaría las respuestas cualquiera que fuera el contexto.

Por lo que se refiere a la pregunta 3, cuyo objetivo se explicitaba en un apartado anterior correspondiente a la elaboración del cuestionario, en la tabla VII se pueden observar los resultados porcentuales correspondientes a la misma (se expresan de forma abreviada y en el mismo orden en que se presentaban en la pregunta).

La alternativa que sugiere una relación más amplia entre microbios y alimentos es elegida en mayor porcentaje que las demás, siendo mayor éste, como era de esperar, en $7^{\circ}$ curso. Las otras dos alternativas microbiológicas que representan ideas restringidas (ya sea por el antropothorfísmo, ya sea por el estudio del suelo) son elegidas en menor porcentaje y éste se reduce en un $50 \%$ al pasar de $4^{\circ}$ curso a $7^{\circ}$ curso, aunque gran parte de esta reducción no se traduce en un incremento de respuestas correctas, sino del grupo de respuestas «No sé». Pero lo más sorprendente ha sido que el esquema ensayado «Nunca» apenas disminuye en $7^{\circ}$ curso, y también que, en general, su aceptación sea tan baja, sobre todo si lo comparamos con la de las alternativas que en las preguntas 1 y 2 ofrecían explicaciones no microbiológicas ( Sin causa» y «Factores físicos o químicos»).
Todo parece indicar que la terminología familiar utilizada en esta $3^{a}$ pregunta desencadena un mayor número de respuestas correctas. Aparentemente estaríamos ante unos alumnos que conocen la relación alimentos-microbios en mayor medida de lo que pudiera deducirse del análisis de las preguntas 1 y 2 , es decir, del análisis de lo que ellos piensan sobre dos efectos concretos de esa relación. Pero también es posible que el lenguaje cotidiano limite los términos en que se establecen esas relaciones. Es posible, por ejemplo, que el término «comer» sirva simplemente para explicar la desaparición del alimento o quizá otras percepciones; pero obviamente parece que no, en este caso al menos, para explicar percepciones como las que suponen la fermentación o el moho. En otras palabras, que los alimentos sirvan de "comida» para los microbios o que sean «atacados" por los microbios parece bastante aceptado; pero existe una muy baja aplicación de ese concepto para explicar los fenómenos producidos o que puede producir esa relación (recordemos, del análisis de las preguntas anteriores, que la fermentación y la formación de moho eran procesos para los que la explicación "Sin causa» revelaba cierta consistencia).

Sería preciso investigar sobre la hipótesis de que los ahumnos, al tratarse de fenómenos inaccesibles a la percepción, utilizan analogías para su comprensión, analogías que ellos mismos activan y que también son puestas a su alcance, a veces por los profesores para facilitar la enseñanza, a veces por el ambiente social y especialmente por los medios de comunicación (dibujos animados de microbios con boca y ojos, soldados que atacan...); y estas analogías, con su correspondiente terminología, facilitan la introducción y comprensión de los conceptos, pero de un modo que, al no ser exactamente científico, dificulta más tarde de forma importante la aplicación de los mismos.

Cuestión 2. ¿Con qué procesos familiares relacionan los alumnos el concepto de fermentación.

En la tabla VIII se puede observar, en expresión porcentual, la elección de procesos o situaciones que los alumnos relacionan con fermentación, teniendo en cuenta que se les pedía que señalasen tres de entre los siete que figuraban en la pregunta (en esta tabla se expresan de forma abreviada y en el mismo orden en que figuraban en la pregunta 4 del cuestionario).

Teniendo en cuenta que la probabilidad de señalar al azar cada opción estaría en torno al $43 \%$, en $4^{\circ}$ curso se observa una desviación positiva en las asociaciones de «infección de heridas» $y$ «hervir líquidos», dos ideas que habían sido muy utilizadas en preguntas abiertas y que pensamos que pueden estar muy relacionadas con el uso del lenguaje, ya que el término «fermentación» se usa a menudo en el ámbito cotídiano con acepciones distintas de la científica. A este respecto, así como en la asociación con «Infección de heridas», ambos cursos resultan diferentes (CHI-C $=10,6 ; \mathrm{p}<0,01$, para $\mathrm{I}$ grado de libertad), en la relación fermentación/ebullición, no existe diferencia significativa entre ellos, siendo todavía una asociación común en $7^{\circ}$ curso. 
TABLA VIII

Pregunta 4

\begin{tabular}{|l|c|c|}
\hline Procesos & $4^{\circ}(\mathrm{n}=145)$ & $7^{\circ}(\mathrm{n}=184)$ \\
\hline Infec. herid. & $58 \%$ & $34 \%$ \\
\hline Elab. Pan & $32 \%$ & $46 \%$ \\
\hline Elab. Vino & $41 \%$ & $65 \%$ \\
\hline Crec. Plantas & $40 \%$ & $27 \%$ \\
\hline Huesos & $33 \%$ & $16 \%$ \\
\hline Etullicion & $61 \%$ & $50 \%$ \\
\hline Elab. Yogur & $24 \%$ & $60 \%$ \\
\hline
\end{tabular}

En cuanto a las relaciones establecidas correctamente, hay que decir que la elaboración del vino ha sido la de mayor porcentaje en ambos cursos, y que las asociaciones correctas se incrementan notablemente al pasar de $4^{\circ}$ a $7^{\circ}$ curso. En este sentido, ambos cursos podrían considerarse similares estadísticamente en cuanto a las relaciones establecidas entre fermentación/elaboración del pan, pero distintos en cuanto a las relaciones establecidas para la elaboración del vino $(\mathrm{CHI}-\mathrm{C}=10,6 ; \mathrm{p}<0,01)$ y de la leche $(C H I-C=25 ; p<0,001)$.

$Y$ finalmente, si entendemos por uso totalmente correcto del término saber reconocer los tres procesos con los que se le puede relacionar, hay que decir que sólo un $5 \%$ de alumnos de $4^{\circ}$ curso y un $21 \%$ de $7^{\circ}$ curso han señalado simultáneamente las tres opciones correctas

\section{CONCLUSIONES}

Los alumnos, a pesar de que conocen que los microorganismos pueden vivir a expensas de los atimentos, muestran escasa capacidad para reconocer causas microbiológicas en el origen de algunas transformaciones alimentarias. Para interpretar estos cambios, utilizan esquemas conceptuales alternativos que revelan cierta consistencia individual, como es el caso de aquéllos que interpretan las transformaciones como un proceso espontánco e intrínseco del alimento, o también los que interpretan que estos cambios surgen debido únicamente a causas físicas o químicas.

Aunque en $4^{\circ}$ curso (9-10 años) los niños poseen un conocimiento biológico que, como sugiere Carey (1985) ha sido recientemente conseguido y reorganizado, $y$ puede considerarse prematura una correcta aplicación de conceptos, el hecho de que las explicaciones microbiológicas no aumenten significativamente en $7^{\circ}$ curso parece, sin embargo, reclamar un mayor esfuerzo por parte de los profesores para que ambos conceptos ( $m i$ crobios y transformaciones) no permanezcan desliga. dos o, como señala Harlen (1989), como «ideas que flotan libremente». Es importante que los profesores promuevan experiencias que abran nuevas posibilidades a los conceptos recientemente adquiridos y que, a la vez, los relacionen con otros que los niños ya tienen.

Por otro lado, una buena red de experiencias-ideas, en el sentido señalado por Harlen (1989), exigiría que los alumnos poseyeran un concepto de contaminación biológica del aire para poder conectar con la contaminación de los alimentos. Pero, en el estudio del aire, creemos que tanto profesores como libros de texto tienen muy en cuenta las causas de contaminación química y se olvidan de la bacteriológica. No puede extrañarnos, por tanto, que, cuando los niños abandonan a partir de $4^{\circ}$ curso las explicaciones de transformación de los alimentos como cambio espontáneo, busquen habitualmente explicaciones mecanicistas o físico-químicas.

En este esfuerzo por relacionar ambos conceptos, no debe desdeñarse el uso de las analogías en ambos cursos (Duit, 1991; Brown, 1993), pero siempre sin olvidar que, en la creación de esquemas por transferencia desde otro dominio, corremos el riesgo de utilizar elementos de éste que no se correspondan con el concepto de estudio y que podrían dificultar, por tanto, la aplicación del mismo.

Por último, en cuanto a la fermentación y con respecto al problema semántico detectado, pensamos que sería importante en estos niveles evitar asociaciones incorrectas que, como señalan diversos autores, pueden ser fuente de problemas en el posterior aprendizaje. Llorens et al. (1984), entre otros, demuestran la relación entre el uso de terminología científica y un mejor nivel de adquisición de conceptos.

\section{AGRADECIMIENTOS}

Los autores agradecen a la Xunta de Galicia (Conselleria de Educación), la fínanciación del Proyecto XUGA29001A91, del cual forma parte la investigación presentada en este trabajo. Agradecen también a M. Pilar Iiménez Aleixandre sus comentarios y sugerencias, de gran utilidad para la redacción de este artículo.

\section{NOTA}

' Se referían diversos conceptos, para que se indicasen los que se trataban en cada curso, relativos a los siguientes aspectos: adquisición de hábitos para una alimentación sana, prevención de enfermedades contagiosas, evolución de los seres vivos, tos microorganismos en los ecosistemas, productos obtenidos a partir de los microorganismos, estudio de la célula y de los microorganismos, estudio de la contaminación. 


\section{REFERENCIAS BIBLIOGRÁFICAS}

ARNAY, J. (1988). Las teorias implicitas sobre el concepto de ser vivo. Tesis doctoral. Universidad de la Laguna.

BARRIO, C. (1990). La comprensión infantil de la enfermedad. Un estudio evolutivo. Madrid: Anthropos.

BLISS, J., MONK, M. y OGBORN, J. (1987). Qualitative Data Analysis for Educational Research. Londres: Croom Helm.

BROWN, D.E. (1993). Refocusing core intuitions: A concretizing role for analogy in conceptual change. Journal of Research in Science Teaching, 30 (10), pp. 1273-1290.

BRUMBY, M. (1982). Student's perceptions of the concept of life. Science Education, 66(4), pp. 613-622.

BRUMBY, M., GARRARD, J, y AUMAN, J. (1985). Student's perceptions of the concept of health. European Journal of Science Education, 7(3), pp. 307-323.

BURGESS, R. (1985). Strategies of Educational Research: Qualitative Methods. Londres: Falmer Press.

CAREY,S. (1985). Conceptual change in childhood. Cambridge, Massachusetts: M.I.T. Press.

CLAXTON, G. (1987). Vivir y Aprender. Madrid: Alianza Ed.

DREYFUS, A. y JUNGWIRTH, E. (1988). The cell concept of 10th graders: curricular expectations and reality. International Journal of Science Education, 10(2), pp. 221-229.

DREYFUS, A. y JUNGWIRTH, E. (1989). The pupil and the Jiving cell: a taxonomy of disfunctional ideas about an abstract idea. Journal of Biological Education, 23(1), pp. 49-55.

DRIVER, R., GUESNE, E. y THIBERGHIEN, A. (1989). Ideas científicas en la infancia y la adolescencia. Madrid: Morata.

DUIT, R. (1991). On the role of analogies and metaphors in learning Science. Science Education, 75(6), pp. 649-672.

ENGEL CLOUGH, E. y DRIVER, R. (1986). A study of consistency in the use of student's conceptual frameworks across different task contexts. Science Education, 70:4), pp. 473-496.

GILBERT, J.,OSBORNE, R. y FENSHAM,P. (1982). Children's science and its consequences for teaching. Science Education, 66 (4), pp. 623-633.

HARLEN,W. (1989). Enseñanza y aprendizaje de la ciencia, Madrid: Morata-MEC.
JIMÉNEZ, M.P. (1990). Los esquemas conceptuales sobre la selección natural: análisis y propuestas para un cambio conceptual. Tesis doctoral: Universidad Complutense.

LUCAS, A., LINKE, R. y SEDGWICK, P. (1979). Schoolchildren's criteria for «alive»: a content analysis approach. The Journal of Psychology, 103, pp. 103-112.

LLORENS, J., JAIME DE, M. y LLOPIS, R. (1989). La función del lenguaje en un enfoque constructivista del aprendizaje de las ciencias. Enseñanza de las Ciencias, 7(2), pp. 111-119.

MONK, M. (1990). A genetic epistemological analysis of data on children's ideas about DC electrical circuits. Research in Science and Technological Education, 8(2), pp. 133-143.

NAGY, M.H. (1951). Children's ideas on the origin of illness. Health Education Journal, 9, pp. 6-12.

NAGY, M.H. (1952). Children's ideas on the activity of germs. Health Education Journal, 10, pp. 15-20.

NAGY, M.H. (1953). The representation of germs by children. Journal of Genetic Psychology, 83, pp. 227-240.

NOVAK, J. y GOWIN, D. (1988). Aprendiendo a aprender. Barcelona: Martínez Roca.

O-SAKI, K y SAMIRODEN,W. (1990). Children's conceptions of "living" and "dead". Journal of Biological Education, 24(3), pp. 199-207.

OSBORNE, R. (1980). Some aspects of student's views of the world. Research in Science Education, 10, pp.11-18.

POZO, J.I. (1987). Aprendizaje de la ciencia y pensamiento causal. Madrid: Visor.

POZO, J., PÉREZ, M., SANZ, A. y LIMÓN, M. (1992). Las ideas de los alumnos sobre la ciencia como teorías implícitas, Infancia y Aprendizaje, 57, pp. 3-2

SALTIEL, E. y VIENNOT, L. (1985). ¿Qué aprendemos de las semejanzas entre las ideas históricas y el razonamiento espontáneo de los estudiantes. Enseñanza de las Ciencias, 3(2), pp. 137-144.

SHAYER, M. y ADEY, P. (1984). La ciencia de enseñar ciencias. Desarrollo cognoscitivo y exigencias del curriculo. Madrid: Narcea.

TREAGUST, D. (1988). Development and use of diagnostic tests to evaluate students' misconceptions in Science. International Journal of Science Education, 10(2), pp. 159-169. 


\section{APÉNDICES}

\section{APÉNDICE 1}

1. Alguien compró una barra de pan recién hecho y la dejó encima del estante de la cocina. Cuando después de 8 días se acordó de ella, se la encontró como se puede ver: con una pelusa blanca y puntitos negros.

a) ¿Qué piensas que es esa pelusa? ¿De qué está hecha?

b) ¿Cómo es que primero no la había? (tardó varios días en aparecer). ¿De dónde crees que procede? POR FAVOR, explica tus contestaciones.

c) ¿Cómo guardarías tú la barra de pan recién comprada para que no ocurra eso? (suponte que no tienes nevera ni congelador). POR FAVOR, explica tus contestaciones.

2. Suponte un juego entre dos, por ejemplo tú y otro amigo, en el que se tienen fichas con letras del abecedario. Se juega, y salen las siguientes fichas:

\section{$|\mathrm{M}| \quad|E| \quad|A| \quad|T| \quad|E| \quad|R| \quad|N||F|$}

Hay que conseguir en un tiempo máximo de dos minutos formar la palabra más larga posible. Tu amigo forma la palabra REMAN, y tư formas FERMENTA. Sin embargo, tu amigo se enfada porque piensa que FERMENTA es una palabra que no existe.

- ¿Cómo le convencerías del significado de esa palabra y le pondrías algún ejemplo de cuando se utiliza?

\section{APÉNDICE 2}

- «Las cosas se pudren solas.» $\left(4^{\circ} \mathrm{EGB}\right)$.

- «El moho procede de la descomposición de los ingredientes del pan, que se pudren con el tiempo.» ( $\left.7^{\circ} \mathrm{EGB}\right)$.

- «Es moho. Es polvo del aire que se deposita con la humedad.» (4 $\left.4^{\circ} \mathrm{EGB}\right)$.

- «Es moho, que está formado por la humedad del pan.» ( $\left.7^{\circ} \mathrm{EGB}\right)$.

\section{APÉNDICE 3}

1. El yogur se hace transformando la leche. Este cambio es producido por:

- Seres vivos a los que les gusta la leche más ácida.

- No lo producen seres vivos, ya que la leche cambia ella sola.

- Seres vivos que estaban en la leche y se multiplicaron.

- No lo producen seres vivos, sino los gases del aire.

- No lo sé.

- Por otras cáusas. (Explica.)

2. Algunos quesos cuando se estropean se ponen de color verde por fuera. Para que esto ocurra hace falta que:

- No hace falta nada, ya que el queso se estropea sólo al pasar el tiempo.

- Hace falta solamente humedad.

- Hace faita que caigan en el queso seres vivos de fuera.

- Hace falta que a los seres vivos les atraiga el olor del queso.

- No lo sé.

- Hacen falta otras cosas. (Explica.) 


\section{Algunos alimentos pueden ser atacados por microbios cuando:}

- Los microbios se depositan en ellos y les damos tiempo a comérselos.

- Los microbios se dan cuenta de que los alimentos están abandonados.

- Los alimentos caen sobre la tierra, ya que sólo allí están los microbios que los atacan.

- Nunca, ya que los microbios no atacan los alimentos.

- No sé cuando.

- En otros casos. (Explica.)

4. Señala, de las siguientes situaciones, tres que a títe parezcan que tienen relación con la FERMENTACIÓN. (Marca solo las tres respuestas que te parezcan más relacionadas.)

- Infección de heridas.

- Elaboración del pan.

- Elaboración del vino.

- Crecimiento de las plantas.

- Roturas de huesos.

- Hervir líquidos.

- Hacer yogur. 
